\title{
Stimulus pre-exposure and instrumental learning
}

\author{
JAMES E. ACKIL AND ROGER L. MELLGREN, INDIANA \\ UNIVERSITY, Bloomington, Ind. 47401
}

Rats were given 0,5 , or 60 pre-exposures (PE) of a tone prior to their learning an active avoidance response to that tone. The $60 \mathrm{PE}$ group learned significantly slower than the $O$ and $S P E$ groups which did not differ from each other. The results confirm and extend to instrumental learning the phenomena of "latent inhibition," which was discussed in terms of two possible explanations-competing responses and attention-mechanisms.

Numerous studies have shown that repeated presentation of a stimulus (e. g., tone) results in relatively systematic changes in EEG activity, other physiological changes (e. g., heart rate, GSR response), and also overt attentional behavior (Sharpless \& Jasper, 1956; Morrell, 1961). The general result is to decrease the various response measures with continued stimulus exposure. Although habituation to a stimulus is well established, the behavioral ettects of habituation in a learning situation have only recently been studied.

Lubow and his associates have found that unreinforced preexposure (PE) of a to-be-conditioned stimulus results in a dramatic decrease in the rate of conditioning to that stimulus (latent inhibition) (Lubow \& Moore, 1959; Lubow, 1965; Chacto \& Lubow, 1967). The procedures used in all these studies were those of classical conditioning, using shock in sheep, goats, and rats. A similar result has been found in a CER situation (Carlton \& Vogel, 1967), where the stimulus paired with unavoidable shock was presented unpaired with shock for one group and not presented until the shock trial for the other group. Suppression ratios showed a marked difference between the groups, with the PE group showing much less suppression than the non-PE group. A similar result has also been found when PE involves the two stimuli (S+, S-) to be used in a discrimination problem. In that study reinforcement was also given during the PE trials, so that the situation was not formally the same as the previous studies, but conceptually similar, with the same decrement due to PE (Mellgren \& Ost, 1968). The present study was designed to extend the above findings to the acquisition of an instrumental response in the rat.

It has also been noted in electrophysiological studies that measures such as EEG "arousal" (Grastyan, 1961), and "vigilance" (Lyman, 1956), increase for a few trials and then begin to habituate. It was felt that a few stimulus exposures might facilitate learning, and for this reason a $5 \mathrm{PE}$ group was included.

Method. Male hooded rats ( $\mathrm{N}=21), 120$ to 150 days old, were housed three to a cage with water and food available ad lib. They were randomly divided into three equal groups. A shuttle box of $1 / 2$ in. plywood, painted black, was used for both the PE and training phases of the experiment. The box was 36 $x 8$ in. and 18 in. in height. The front of the box was clear Plexiglas, and the floor was made of 69 brass rods, $3 / 8$ in. in diameter spaced at intervals of $1 / 2$ in. center-to-center. The box was illuminated with a $6-W$ bulb located at the top-center of the box. A 5-in. speaker at the top-center of the box delivered a $1000 \mathrm{cps}$ tone at approximately $80 \mathrm{~dB}$, which was used as the CS. Scrambled shock of $.4 \mathrm{~mA}$ was delivered via the bars to the feet of $\mathrm{S}$.

Each $S$ was run in one continuous session consisting of a $45 \mathrm{~min}$ PE phase and an acquisition phase which lasted until $S$ made 8 of 10 avoidance responses. All groups were in the shuttle box for the $45 \mathrm{~min}$ PE period. Group $5 \mathrm{PE}$ received five tone presentations of $5 \mathrm{sec}$ duration, $45 \mathrm{sec}$ apart, during the last $4 \mathrm{~min}$ of $\mathrm{PE}$, Group $60 \mathrm{PE}$ received 60 tones of $5 \mathrm{sec}$ duration $45 \mathrm{sec}$ apart during the entire $45 \mathrm{~min}$, and Group $\mathrm{O} P E$ had no tone presentations. Training commenced immediately following the $45 \mathrm{~min}$ PE period, with tone signalling a shock in $5 \sec ($ ITT $=45 \mathrm{sec}$ ) unless $S$ shuttled from the side he was on at the tone onset to the other side of the box (avoidance). If $S$ did not shuttle in $5 \mathrm{sec}$, tone remained on and shock was delivered until $S$ escaped by moving to the other side of the box. A response during the tone or tone and shock immediately resulted in their offset.

Results. A significant effect for the three PE conditions was revealed by an analysis of number of trials to the acquisition criterion. The overall $F$ was significant beyond the .05 level $(F=3.98$, $\mathrm{df}=2 / 18$ ). The differences were due to the $60 \mathrm{PE}$ group differing from 0 and $5 \mathrm{PE}$ groups, mean number of trials to criterion being $40.6,27.6$, and 27.0 , respectively. A planned comparison was car- ried out between the 0 and 60 PE groups, which confirmed the obvious finding that $\mathrm{PE}$ significantly retarded acquisition of the instrumental response to that stimulus $(F=5.70, \mathrm{df}=1 / 18$, $p=.03$ ). Total errors (escapes) were also analyzed and showed substantially the same thing as trials to criterion, except to a lesser extent. Trial of first avoidance was also analyzed, but showed no significant trends, although the 5 PE group was slightly superior in this measure, with a mean of 10.9 as compared to 13.0 and 13.3 for the 0 and $60 \mathrm{PE}$ groups, respectively.

Discussion. The results establish that PE of a stimulus significantly retards acquisition of an instrumental response to that stimulus. Lubow (1965) has suggested two possible explanations of this effect, competing responses and attentional decrements. Presumably, a competing response explanation would dictate that during $\mathrm{PE}$, responses are conditioned to the stimulus, and these responses interfere with the association of a new response in training to that stimulus. An explanation involving attentional mechanisms, on the other hand, would dictate that during PE S learns that the stimulus has no signal value. Thus the effect of PE would be a type of "conditioned ignorance." Both of these explanations assume that some sort of learning has occurred during PE and that this interferes with subsequent learning. In this light, attentional deficits may be thought of as a special case of a competing response, since the concept of a competing response is left insufficiently defined. Both of these explanations would predict that the deficit found with $P E$ would be due to an inability to make the appropriate avoidance response. Competing responses would require the animal to extinguish any competing response to the stimulus in order to make the correct avoidance response. The attentional explanation would require the animal to learn to attend to the stimulus. Both of these explanations would make the prediction that trial of first avoidance, which may be taken as a rough measure of the extinction of competing responses, or a measure of attending to the stimulus, to be greater for the $60 \mathrm{PE}$ group than the O PE group. Since this was not the case, the conclusion must be that either these notions are not sufficiently refined or trial of first avoidance is not a sensitive enough measure.

The $5 \mathrm{PE}$ group did not differ from the $\mathrm{O}$ PE group, indicating that a small number of PEs is not sufficient to produce the inhibitory effect. The absence of a possible facilitating effect suggests that the influences of increased vigilance or arousal are not evident in this conditioning situation.

Two further aspects of the data of this study and others (e. g., Chacto \& Lubow, 1967) are worthy of note. One is the rather large intersubject variability of the PE group that was observed in this experiment, and those of Lubow and his associates. Typically, some Ss show a very dramatic deficit, while others show none. What variables control the effect are unknown at the present time and are in need of investigation. A second aspect to note is the common practice in neurophysiological studies of learning of habituating a CS prior to training so that physiological response to the stimulus is at some base-point at the start of training. In light of the inhibitory effects that have been found with stimulus PE, this practice may give a grossly distorted view of the usual conditioning process.

\section{REFERENCES}

CARLTON, P. L., \& VOGEL, J. R. Habituation and conditioning. J. comp. physiol Psychol, 1967,63, 348-351.

CHACTO, C., \& LUBOW, R. E. Classical conditioning and latent inhibition in the white rat. Psychon. Sci, 1967,9, 135-136.

GRASTYAN, E. The significance of the earliest manifestations of conditioning in the mechanism of learning. In Fessard, A., Gerard, R. W., Konovski, J. and Delafresnaye, J. F. (Eds.), Brain mechanisms and learning. Springfield: Charles C. Thomas, 1961. Pp. 231-242.

LUBOW, R. E. Latent inhibition: effects of frequency of nonreinforced pre-exposure of the CS. J. comp. physiol. Psychol, 1965, 60, 454-457.

LUBOW, R. E., \& MOORE, A. V. Latent inhibition: the effect of nonreinforced pre-exposure to the conditioned stimulus. J. comp. physiol. Psychol, 1959, $52,415-419$.

LYMAN, B. Signal vigilance level and conditioned avoidance acquisition in the goat.Dissert. Abstr., 1956, 1725-1726.

MELLGREN, R. L., \& OST, J. W. P. Transfer of Pavlovian discrimination training to an operant discrimination. Paper read at Midwest Psychol. Assoc. Conv., Chicago, 1968.

MORRELL, F. Electrophysiological contribution to the neural basis of learning. Physiol Rev., 1961, 41, 433-444.

SHARPLESS, S., \& JASPER, H. H. Habituation of the arousal reaction. Brain, $1956,79,655-680$.

1. The research reported here was supported in part by Public Health Service Grant HD00951-05 to J.W.P. ()st. 